\title{
Intoxicação por Erythroxylum deciduum (Erythroxylaceae) em ovinos $^{1}$
}

\author{
Edson Moleta Colodel ${ }^{2}$, Anderson Luis Seitz², Milene Schmitz², Mauro Riegert \\ Borba² $^{2}$ Djeison Lutier Raymundo² e David Driemeier ${ }^{2}$
}

\begin{abstract}
Colodel E.M., Seitz A.L., Schmitz M., Borba M.R., Raymundo D.L. \& Driemeier D. 2004. [Poisoning by Erythroxylum deciduum (Erythroxylaceae) in sheep.] Intoxicação por Erythroxylum deciduum (Erythroxylaceae) em ovinos. Pesquisa Veterinária Brasileira 24(3):165-168. Setor de Patologia Veterinária, Depto Patologia Clínica Veterinária, Faculdade de Veterinária, Universidade Federal do Rio Grande do Sul, Av. Bento Gonçalves 9090, Cx. Postal 15094, Porto Alegre, RS 91540-000, Brazil. E-mail: moleta@ terra.com.br.

The epidemiological, clinical and pathological aspects of natural poisoning caused by Erythroxylum deciduum fruits in sheep are reported. The outbreak occurred from January to March of 2004, in Lagoa Vermelha county, state of Rio Grande do Sul, Brazil. The poisoning was experimentally reproduced by oral administration of $E$. deciduum fruits to 5 sheep. Three of them developed neurological signs and died. Poisoning was caused with a single dose of $60 \mathrm{~g} / \mathrm{kg}$ or when the dosage was fractioned into at least 4 doses of $17 \mathrm{~g} / \mathrm{kg}$ given every 12 hours. The main clinical signs in natural and experimentally poisoned sheep were ataxia, hyperexcitability and muscular tremors which where more pronounced when the animals were moved. At the final stage, dyspnea with abdominal breathing and cyanosis was observed. The most significant alterations found at necropsy were pronounced edema, lung congestion and presence of the fruits or seeds of $E$. deciduum in the ruminal content of all animals. Histologically, except edema and pulmonary congestion, there were no other significant changes observed.
\end{abstract}

INDEX TERMS: Poisonous plants, plant poisoning, experimental plant poisoning, Erythroxylum deciduum, Erythroxylaceae, sheep.

RESUMO.- Este trabal ho descreve os aspectos epidemiológicos, clínicos e patológicos da intoxicação natural pelos frutos de Erythroxylum deciduum ("cocão") em ovinos, que ocorreu de janeiro a março de 2004, no município de Lagoa Vermelha, RS. A doença foi reproduzida pela administração dos frutos de $E$. deciduum por via oral a 5 ovinos. Destes, três adoeceram e morreram. Os frutos de E. deciduum foram tóxicos em dose única de $60 \mathrm{~g} / \mathrm{kg}$ ou quando fracionado em pelo menos 4 doses de $17 \mathrm{~g} /$ $\mathrm{kg}$ a cada 12 horas. Os principais sinais clínicos nos animais intoxicados natural e experimentalmente, foram neurológicose caracterizados por ataxia, hiperexcitabilidade e tremores musculares que se pronunciavam durante o manejo. Próximo à morte, os ovinos apresentavam dispnéia com respiração abdominal

\footnotetext{
${ }^{1}$ Recebido em 16 de junho de 2004.

Aceito para publicação em 28 de junho de 2004.

2 Setor de Patologia Veterinária, Departamento de Patologia Clínica Veterinária, Faculdade de Veterinária, Universidade Federal do Rio Grande do Sul (UFRGS), CX. Postal 15094, Porto Alegre, RS 91540-000. E-mail: moleta@ terra.com.br
}

e cianose. Na necropsia as alterações mais importantes, em 6 casos de intoxicação espontânea e os 3 ovinos experimentalmente intoxicados, foram edema e congestão pulmonar acentuada e a presença de frutos ou sementes de E. deciduum no conteúdo ruminal. Histologicamente, exceto edema e congestão pulmonar, não foram encontradas outras alterações significativas.

TERMOS DE INDEXAÇÃO: Plantas tóxicas, intoxicação por planta, intoxicação experimental por planta, Erythroxylum deciduum, Erythroxylaceae, ovinos.

\section{INTRODUÇÃO}

0 gênero Erythroxylum, da família Erythroxylaceae, compreende cerca de 250 espécies (Cronquist 1981). Deste gênero, Erythroxylum coca é conhecido como fonte natural da cocaína. No Rio Grande do Sul ocorrem nove espécies, incluindo Erythroxylum deciduum St. Hil. que popularmente é conhecido como "cocão". E. deciduum é uma arvore que alcança até $8 \mathrm{~m}$ de altura e ocorre especialmente em áreas tropicais. Trata-se de uma espécie pioneira, encontrada em beira de matas, vassourais e cerrados. Floresce de agosto a janeiro e os frutos são observa- 
dos de setembro a fevereiro (Lorenzi 2002). Históricos de intoxicação natural em ovinos por essa planta foram recentemente relatados (Motta et al. 2004).

0 objetivo desse trabalho é descrever os achados clínicos e patológicos das intoxicações natural e experimental por Erythroxylum deciduum em ovinos no Estado do Rio Grande do Sul.

\section{MATERIAL E MÉTODOS}

\section{Histórico clínico e classificação botânica}

Os históricos clínicos da intoxicação natural em ovinos, que ocorreram nos meses de janeiro a março de 2004, foram obtidos junto ao proprietário e aos veterinários da propriedade afetada, no município de Lagoa Vermelha, Rio Grande do Sul. Frutos de Erythroxylum deciduum St. Hil. (Fig. 1) foram coletados em fevereiro de 2004, nesta propriedade. Amostras botânicas foram identificadas no Instituto de Ciências Biológicas, Universidade Federal de Minas Gerais.

\section{Estudo experimental}

Foram utilizados 5 ovinos, adultos, mestiços, clinicamente sadios (Quadro 1). Durante o experimento os animais foram mantidos individual mente em baias de alvenaria e receberam água ad libitum, ração comercial para ovinos e feno de alfafa. Imediatamente antes do experimento os frutos de Erythroxylum deciduum foram misturados em água, triturados em liquidificador e fornecidos por via oral, com auxilio de uma seringa cortada na parte anterior. Posteriormente foram realizados exames clínicos periódicos avaliando-se, temperatura corporal, movimentos ruminais, freqüências

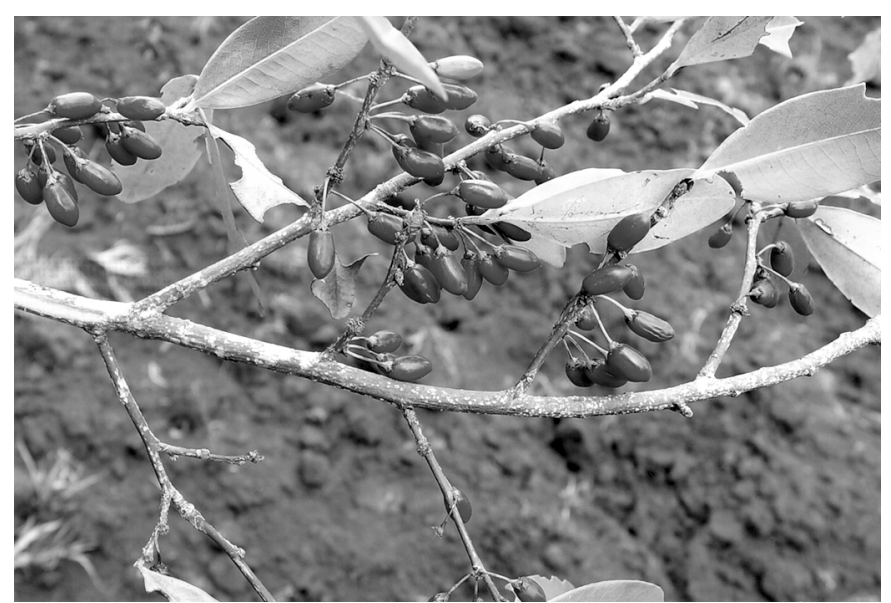

Fig. 1. Galho de Erythroxylum deciduum em frutificação, no mês de fevereiro, Lagoa Vermelha, RS. respiratória e cardíaca e avaliação da movimentação espontânea e forçada.

\section{Estudo patológico}

Foram necropsiados 6 ovinos naturalmente intoxicados, no município de Lagoa Vermelha, RS, e 3 ovinos experimentalmente intoxicados pelos frutos de E. deciduum no Setor de Patologia Veterinária da Faculdade de Veterinária, Universidade Federal do Rio Grande do Sul. Durante a necropsia fragmentos de fígado, rim, pulmão, coração, diferentes segmentos gástricos e intestinais e o sistema nervoso central foram fixados em solução de formalina tamponada $10 \%$ Amostras destes tecidos, incluindo cortes transversais do sistema nervoso central no corpo estriado sob o quiasma óptico, tálamo sob o corpo mamilar, mesencéfalo sobre os colículos caudais, cerebelo sobre o braço da ponte, medula oblonga sobre o óbex, medula espinhal nas intumescências cervical e lombar foram rotineiramente processadas e coradas com hematoxilina-eosina (Prophet et al. 1992) para estudo microscópico. Amostras do conteúdo ruminal, fígado e rim dos animais necropsiados foram submetidos ao processo de preparação por solventes orgânicos e o extrato resultante foi analisado por método de cromatografia em camada delgada (Narayanaswami et al. 1974) para pesquisa de organofosforados.

\section{RESULTADOS}

\section{Intoxicação natural}

Em uma propriedade, no município de Lagoa Vermelha, RS, no mês de janeiro de 2004 de um lote de 70 ovinos, adoeceram 17 (13 Suffolk, 4 lle de France). Os animais apresentaram alterações clínicas caracterizadas por dificuldade para acompanhar o rebanho, andar lento com os membros rijos, dismetria, quedas em decúbito lateral com tetania e cabeça voltada para traz ou para o flanco. Apresentaram também dispnéia com respiração abdominal, intensa salivação, cianose e morte em evoluções clínicas de 8 a 24 horas. As alterações clínicas se exacerbavam quando os animais eram manejados. Dois animais foram encontrados mortos e outros dois morreram durante o tratamento com polivitamínico e foram necropsiados por veterinários que atendiam a propriedade. Não foram relatadas alterações macroscópicas. Os demais ovinos que apresentaram alterações clínicas se recuperaram. A doença ocorreu em piquetes de pastagem natural ou de restevas de lavoura de batata circundadas com mata ciliar e coincidia com o período de queda dos frutos de Erythroxylum deciduum. Notou-se que os ovinos consumiam com avidez os frutos caídos, misturados à pastagem. Os piquetes onde ocorria esta árvore foram isolados, impedindo-se a entrada dos animais. Em março de 2004, aproximadamente 20

Quatro 1. Intoxicação experimental por Erythroxylum deciduum em ovinos

\begin{tabular}{ccccccccc}
\hline $\begin{array}{c}\text { Ovino } \\
\text { no. }\end{array}$ & $\begin{array}{c}\text { Peso } \\
(\mathrm{kg})\end{array}$ & $\begin{array}{c}\text { Idade } \\
\text { (meses) }\end{array}$ & $\begin{array}{c}\text { Dose } \\
(\mathrm{g} / \mathrm{kg})\end{array}$ & $\begin{array}{c}\text { Número } \\
\text { de doses }\end{array}$ & $\begin{array}{c}\text { Intervalo } \\
\text { entre do- } \\
\text { ses }(\mathrm{h})\end{array}$ & $\begin{array}{c}\text { Início dos } \\
\text { sinais clí- } \\
\text { nicos }(\mathrm{h})\end{array}$ & $\begin{array}{c}\text { Evolução } \\
\text { clínica }(\mathrm{h})\end{array}$ & Desfecho \\
\hline 1 & 50 & 48 & 20 & 3 & 12 & 25 & 1 & Morte \\
2 & 30 & 12 & 17 & 4 & 12 & 28 & 18 & Morte \\
3 & 27,5 & 24 & 9 & 14 & 12 & Sa & Sa & Sa \\
4 & 17 & 24 & 60 & 1 & 0 & 7 & 9 & Morte \\
5 & 30 & 12 & 30 & 1 & 0 & Sa & Sa & Sa
\end{tabular}

a Sem alterações clínicas. 


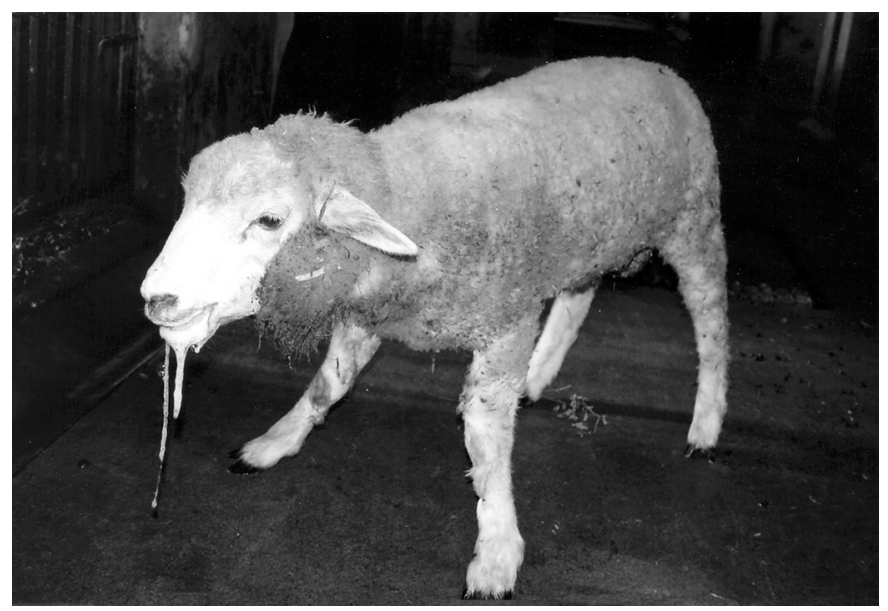

Fig. 2. Ovino experimentalmente intoxicado pelos frutos de Erythroxylum deciduum, em posição de cavalete, com pescoço distendido e com intensa sialorréia.

dias após o termino da queda dos frutos de E. deciduum, outros 12 ovinos adoeceram (11 Suffolk e 1 Ile de France) e 7 animais morreram (6 Suffolk e 1 Ile de France), após pastorearem nos mesmos piquetes que causaram problemas em janeiro. Constatou-se que dos frutos de Erythroxylum deciduum caídos, ainda em bastante quantidade, permaneceram somente as sementes e quando com polpa esta esteve bastante ressecada. Seis animais foram necropsiados por nossa equipe. Não havia alterações macroscópicas significativas. No conteúdo ruminal de todos os ovinos necropsiados havia grande quantidade de sementes de $E$. deciduum. De um lote de aproximadamente 40 bovinos que pastoreavam na mesma área, nenhum foi afetado.

\section{Intoxicação experimental}

Resumo dos aspectos clínicos da intoxicação experimental por Erythroxylum deciduum está relatado no Quadro 1. Dos 5 ovinos que receberam os frutos de E. deciduum, 3 morreram e 2 não apresentaram alterações clínicas. Doses fracionadas a partir de $17 \mathrm{~g} / \mathrm{kg}$ (Ovino 2) e a única de $60 \mathrm{~g} / \mathrm{kg}$ (Ovino 4) causaram a morte dos animais. 0 início dos sinais clínicos variou de 7 (Ovino 4) a 28 horas (Ovino 2) e a evolução clínica foi de 1 (Ovino 1) a 18 horas (Ovino 2) após a administração dos frutos. Os sinais clínicos observados foram predominantemente neurológicos e caracterizados por hiperexcitabilidade, ataxia, dismetria com hipermetria, tiveram tremores musculares generalizados e balançavam a cabeça lateralmente (Fig. 2). Principalmente quando movimentados caminhavam com os membros estendidos, em pequenos saltos. Com evolução do quadro, durante a movimentação, os ovinos caiam em decúbito lateral, permanecendo nesta posição por períodos de até um minuto, tentando se levantar, sem êxito. A dificuldade respiratória se acentuava próximo a morte dos animais, ocorrendo marcada dispnéia com respiração abdominal, acentuada cianose e fluxo de conteúdo espumoso pela cavidade oral e nasal.

\section{Achados patológicos}

Os principais achados nos animais necropsiados, do surto natural e nos experimentalmente intoxicados, foram o marcado edema pulmonar, hemorragias nas serosas, principalmente pleura visceral e no conteúdo do rúmen havia grande quantidade de sementes e frutos inteiros de Erythroxylum deciduum. No exame histo patológico não foram encontradas alterações significativas. Não foram constatados resíduos de organofosforado no exame toxicológico.

\section{DISCUSSÃO}

Este estudo demonstra a toxidez dos frutos de Erythroxylum deciduum para ovinos em doses únicas de $60 \mathrm{~g} / \mathrm{kg}$, ou em doses fracionadas de $17 \mathrm{~g} / \mathrm{kg}$. As evidencias clínicas, os achados patológicos, similares aos encontrados nos ovinos intoxicados experimentalmente, indicam que a ingestão espontânea por frutos desta planta seja a causa da mortandade de ovinos no município de Lagoa Vermelha, RS.

Traços de cocaína foram encontrados em folhas do $E$. deciduum (Aynilian et al. 1974), mas o principio tóxico de E. deciduum, responsável pelas alterações clínicas e morte dos ovinos neste experimento, ainda não é conhecido. 0 quadro clínico da intoxicação por E. deciduum deve ser diferenciado da intoxicação por plantas e micotoxinas que causam síndromes tremorgênicas, como as que ocorrem na intoxicação por Claviceps paspali (Riet-Correa et al. 1983), Cynodon dactylon (Riet-Correa 1993), I pomoea asarifolia (Medeiros et al. 2003) e Phalaris angusta (Gava et al. 1999). As intoxicações por C. dactylon ("grama-bermuda") e por metabólitos do fungo C. paspali, que são associadas ao pastoreio de Paspalum dilatatum ("capim-melador") eP. notatum ("grama-forquilha"), ocorrem, no Rio Grande do Sul, principalmente bovinos, mas também foram observados ovinos intoxicados (Riet-Correa 1993). Ipomoea asarifolia ocorre em regiões litorâneas do Brasil, relata-se a intoxicação em caprinos, ovinos e bovinos, na Região Nordeste. Nestes quadros de intoxicação, não ocorrem alterações patológicas significativas. Na intoxicação por Phalarisangusta relatada em bovinos no Estado de Santa Catarina e que também pode afetar ovinos, observa-se coloração cinza-esverdeado principalmente no tálamo e mesencéfalo.

Não se conhece tratamento específico para a intoxicação pelos frutos de E. deciduum em ovinos. Durante nossas observações, constatou-se que a movimentação dos animais precipitava a morte. Diminuição da mortalidade ocorreu quando os animais foram calmamente retirados dos piquetes com problema e mantidos em ambiente sem movimentação, por períodos mínimos de 24 horas.

Agradecimentos.- Ao Dr. Marcos Eduardo Guerra Sobral pela identificação do material botânico.

\section{REFERÊNCIAS}

Aynilian G.H., Duke J.A., Gentner W.A. \& Farnsworth N.R. 1974. Cocaine content of Erythroxylum species. J. Pharm. Sci. 63(12):1938-1939.

Cronquist A. 1981. An Integrated System of Classification of Flowering Plants. Columbia University, New York. 1262p.

Gava A., Sousa R.S., de Deus M.S., Pilati C., Cristani J., Mori A. \& Neves D.S. 1999. Phalaris angusta (Gramineae) como causa de enfermidade neurológica em bovinos no Estado de Santa Catarina. Pesq. Vet. Bras. 19 (1):3538.

Lorenzi H. 2002. Árvores Brasileiras. Vol. 2. Editora Plantarum, Nova Odessa. $368 p$.

Medeiros R.M.T., Barbosa R.C., Riet-Correa F., Lima E.F., Tabosa I.M., Barros 
S.S., Gardner D.R. \& Molyneux R.J. 2003. Tremorgenic syndrome in goats caused by Ipomoea asarifolia in Northeastern Brazil. Toxicon 41: 933-935.

Motta A.C., Severo B.M.A., Barbisan J., Schimidt O., Pereira R.A., Moraes R.B. \& Ostrowki. 2004. Intoxicação por Erythroxylum deciduum St. Hil. (cocão) em ovinos no planalto médio do Rio Grande do Sul. XVI Congresso Estadual de Medicina Veterinária, Passo Fundo, RS. (Resumo em Seção Grandes Animais, Clínica Cirúrgica e Nutrição)

Prophet E.B., Mills B., Arrington J.B. \& Sobin L.H. 1992 Laboratory Methods in Histotechnology. Armed Forces Institute of Pathology, Washington, DC. $279 p$.

Riet-Correa F. 1993. Intoxicação por Cynodon dactylon, p.127-131. In: RietCorrea F., Méndez M.C. \& Schild A.L. (ed.) Intoxicações por Plantas e Micotoxicoses em Animais Domésticos. Editorial Hemisfério Sul do Brasil, Pelotas. 340p.

Riet-Correa F., Schild A.L., Méndez M.C., Tavares A.S. \& Rodrigues J.O. 1983. Intoxicação por Claviceps paspali em bovinos no Rio Grande do Sul. Pesq. Vet. Bras. 3: 59-65. 